\title{
Anesthetic management of tracheo-esophageal fistula
}

\author{
Filiz Uzumcugil \\ Department of Anesthesiology and Reanimation, Hacettepe University School of Medicine, Ankara, Turkey \\ Correspondence to: Filiz Uzumcugil, MD, DESA. Hacettepe University School of Medicine, Department of Anesthesiology and Reanimation, Sihhiye, \\ 06230, Ankara, Turkey. Email: filizuzumcugil@hotmail.com.
}

\begin{abstract}
The anesthetic management of a neonate diagnosed to have tracheoesophageal fistula (TEF) with or without esophageal atresia (EA) is challenging, especially due to abnormally connected airway and esophagus interfering with patency of the airway and compromising ventilation. The anatomical variations regarding this congenital anomaly and the associated anomalies in various systems, determines both the surgical intervention and the anesthetic management. An urgent surgical intervention may be required within the first 48 hours after birth in case of severe respiratory compromise. On the other hand, a staged approach may be preferred including a gastrostomy in case of either the presence of low-birth-weight, isolated EA or more critical co-morbidities. A laryngotracheobronchoscopy is often performed prior to definitive surgery in order to identify the location and size of fistula, as well as, secondary upper airway anomalies. In the preoperative period, airway must be secured, and optimizing the status of the neonate in terms of other system functions should have a high priority. Intraoperatively, regardless of the agents used, the anesthetic management should focus on adequate depth of anesthesia, and adequate ventilation and oxygenation. The coordination between the anesthetist and the surgeon is crucial during the surgery in order to secure adequate ventilation and oxygenation. The associated anomalies should be considered as the main determinants of perioperative mortality and morbidity, hence, anesthetic management should also focus on intraoperative maintenance of preoperatively optimized functions. The analgesic management in the postoperative period is often provided by multimodal analgesic use.
\end{abstract}

Keywords: Airway management; anesthesia; esophageal atresia (EA); neonate; tracheosophageal fistula (TEF)

Received: 07 December 2020; Accepted: 16 March 2021; Published: 25 August 2022.

doi: $10.21037 /$ cets-20-183

View this article at: http://dx.doi.org/10.21037/ccts-20-183

\section{Introduction}

The anesthetic management of a neonate with tracheoesophageal fistula (TEF) is challenging due to many factors including the connection between the airway and the esophagus leading to difficulty in lung ventilation, the associated anomalies most frequently pertaining to cardiovascular system, and the thoracotomy procedure with its potential adverse hemodynamic consequences and complicated postoperative analgesic management. The anatomical variations regarding the anomaly itself mostly determine the type of the anesthetic management and the surgical technique, as well. The varying techniques for anesthetic management mainly focusing on adequate ventilation strategies will be reviewed in this paper.

\section{Preoperative evaluation and management}

The TEF with or without esophageal atresia (EA) is a congenital anomaly associated with various system anomalies. This congenital malformation can be diagnosed antenatally and the delivery can be scheduled earlier ensuring that the benefit of delivery is balanced against a timely delivery (1). The better understanding of outcome and long-term complications following the surgical repair, which have not actually been clear yet, might lead perinatal counseling provide beneficence (1). However, antenatal ultrasonography might be helpful in the presence of EA, whereas, it is not as useful in an H-type TEF (TEF without EA), in which polyhydramnios is not likely (2).

This congenital anomaly is often associated with the 
anomalies in the acronym VACTERL, which is constituted of vertebral anomalies, imperforated anus, congenital heart disease, tracheoesophageal atresia, renal anomalies and limb anomalies (radial atresia). The association between these anomalies, which were reported to exist in approximately $50 \%$ of the cases, has not been identified in terms of its cause. It is known that the EA can be presented as an isolated anomaly, whereas, it is associated with a TEF in $80-85 \%$ of the patients $(3,4)$. The most common anatomical form is the association of a blind proximal esophageal pouch and a distal TEF (Gross Type C-85\%), whereas, the isolated EA (Gross Type A), the association of a proximal TEF and a distal EA (Gross Type B), the presence of both proximal and distal TEF (Gross Type D), and the isolated TEF [Gross Type E (H-Type)] have incidences of $8 \%, 2 \%$, $1 \%$ and $4 \%$, respectively (3).

The diagnosis of the most common form (Gross Type C) can be confirmed at birth, with the inability to advance an orogastric tube for $8-13 \mathrm{~cm}$. In addition to this finding, the presence of gas in the stomach and the orogastric tube curled at the T2-T4 level in the blind esophageal pouch on a 'babygram' may constitute the first signs. The isolated EA without fistula is presented with the absence of gas in the stomach and bowel. The blind esophageal pouch demonstrated in radiographic studies using radiopaque dye may also confirm the diagnosis $(4,5)$. The pediatric anesthetist and the neonatologist often have the chance to be informed by the surgeons or by the obstetric anesthetist either when there is a scheduled birth with possible antenatal diagnosis of TEF with EA or immediately after the birth of a neonate with the anomaly. On the other hand, the less common form of TEF without an associated EA may be difficult to diagnose either prior to or immediately after birth. These neonates and the ones that cannot be diagnosed immediately after birth, often present with excessive secretions, regurgitation leading to respiratory problems during feeding and recurrent pneumonia due to pulmonary aspiration of the feedings (4). Recurrent pneumonia is often the presentation later in life in patients with H-type TEF, as well as, cyanotic spells and choking while feeding $(2,4)$.

\section{Airway and respiratory system}

The urgent surgical intervention indicated according to the type of TEF often planned within the first 48 hours to prevent aspiration, which may cause severe respiratory compromise (3). On the other hand, a staged approach providing time for the growth is often preferred if the neonate is $<1 \mathrm{~kg}$ or there is an isolated EA or there are more critical co-morbidities (5). The staged repair allows the infant to grow so that the distance between the proximal pouch and stomach to become shorter in order to allow an esophageal anastomosis possible. During this period, a gastrostomy tube to provide drainage of stomach and a central catheter to receive parenteral nutrition are required (4). The neonates should be nursed at $30^{\circ}$ head-up inclined position, in prone or lateral, with a $10-\mathrm{F}$ suction tube placed in the blind proximal pouch of esophagus to decrease the pulmonary aspiration of the secretions $(3,4)$. A constant suctioning preoperatively decreases the accumulation of secretions. In an H-type TEF, as recurrent pneumonia may be the presentation later in life, the lungs may have decreased compliance, which may require optimization of respiratory functions prior to the operation (2).

\section{Cardiovascular system}

Echocardiographic evaluation should be considered prior to the operation, because the position of the aortic arch will determine the side of the thoracotomy. In the presence of a normal left-sided aortic arch, the often preferred right thoracotomy can be performed, otherwise surgeons may prefer the left thoracotomy to approach the fistula (5). Aside from the abnormally positioned aortic arch (rightsided aortic arch; $2.5 \%$ ), 30\% of TEF/EA cases may also have associated cardiac anomalies, such as ventricular septal defect, patent ductus arteriosus, tetralogy of Fallot, atrial septal defect and coarctation of aorta, which constitute major factors predicting mortality and adverse events during surgery $(3,5)$.

\section{Gastrointestinal system}

The anomalies regarding gastrointestinal system may also include imperforated anus, malrotation of midgut, pyloric stenosis and duodenal atresia (5).

\section{Musculoskeletal system}

An ultrasonographic evaluation of spine or X-ray imaging may be required to identify any vertebral anomalies (1).

Aside from the evaluation of associated anomalies in the systems and optimizing their functions preoperatively, routine preoperative blood tests and correction of electrolytes accordingly, humidified oxygen therapy, 
warming, infusing glucose-containing maintenance fluids, as well as preparation of blood products all constitute various parts of preoperative management (2).

\section{Intraoperative management}

General anesthesia is frequently planned for these patients. When it comes to a staged repair, awake gastrostomy and then, a retrograde advancement of a Fogarty catheter in order to occlude the distal end of fistula, by using fiberoptic bronchoscope (FOB), can be planned. For the definitive procedure, endotracheal intubation should be planned with the confirmation of tip placement using FOB (5). It is crucial for the anesthetist to have a plan for the scheduled procedure.

The standard monitoring should include the electrocardiogram (ECG), non-invasive blood pressure and peripheral oxygen saturation $\left(\mathrm{SpO}_{2}\right)$. The pulse-oxymeter should be placed considering the preductal and postductal oxygen saturations (5). An arterial line to monitor real-time blood pressure measurement may help throughout the operation especially for patients with cardiac anomalies.

The estimated blood-loss is minimal during this operation. However, two 22-24 G intravenous catheters should be in place, one of them to be used for continuous infusions of glucose-containing solutions at a fixed-rate, while the other to be used for replacements and bolus doses of drugs.

\section{Anestbetic induction and airway management}

The presence of TEF interfering with the patency of the airway requires airway management to secure ventilation. An awake intubation may be a choice with thorough topical anesthesia in hemodynamically stable neonates. However, inhalational anesthetic induction can be preferred ensuring that the spontaneous ventilation is preserved to secure the airway either during endotracheal intubation or during rigid bronchoscopy performed to evaluate the localization and the size of the fistula prior to repair $(4,5)$.

Anesthetic induction can be provided intravenously, by inhalation or by a combination of techniques including topical anesthesia. Above all precautions, it is important to provide an adequate depth of anesthesia for endotracheal intubation if it is indicated. During the anesthetic induction, it has been emphasized that spontaneous ventilation should be preserved in order to minimize the gastric insufflation (5).
The negative pressure generated during spontaneous ventilation diverts the air inflow towards the lungs not the fistula (3). However, the patients with fistulas $\leq 3 \mathrm{~mm}$ in diameter have been reported to be safe with positive pressure ventilation in terms of gastric insufflation leading to respiratory compromise (2). It should be highlighted that after endotracheal intubation peak inspiratory pressure must be kept low (5). Following the induction, neuromuscular blockade may be required to facilitate intubation. The evaluation of the TEF using rigid bronchoscopy can be performed prior to endotracheal intubation. Regardless of the induction technique, the short periods either before the rigid bronchoscopy or endotracheal intubation may require 'gentle bag-mask ventilation' by the anesthetist, which may still result in gastric insufflation, so that, the communication between the surgeon and the anesthetist is quite important at this stage. The laryngotracheobronchoscopy performed prior to definitive surgery using rigid or flexible bronchoscope has been recommended for every neonate with this anomaly (6). Aside from the location and size of the fistula, it has been reported that the associated secondary upper airway anomalies such as laryngeal cleft, laryngomalacia, subglottic stenosis, tracheomalacia and vocal fold paresis, which worth evaluation due to their incidence and their impact on ventilation, as well as, airway management itself (7-9).

Subsequent endotracheal intubation may result in intubation of the fistula despite prior evaluations for its location and size, if the TEF is large (3). Alternative strategies ensuring adequate ventilation include tracheal intubation distal to fistula, which may lead to one-lung ventilation, or occlusion of the fistula using Fogarty catheter. The occlusion of the fistula by using Fogarty catheter can be performed during bronchoscopy (4). Although the catheter would be theoretically directed towards the fistula on the posterior wall of the trachea in supine position, this procedure may compromise adequate ventilation especially in neonates with respiratory distress $(2,4)$. After the placement of the catheter, its balloon is inflated and rigid bronchoscopy is taken out to allow endotracheal intubation using laryngoscope (2). In the presence of a gastrostomy as a component of a staged repair, the retrograde advancement of Fogarty catheter through gastrostomy may allow safer approach to occlude the distal esophagus (4). These catheters may dislodge easily and may also cause damage to the mucosa by its inflated balloon (2). Although the occlusion of the TEF with a catheter allows adequate ventilation, unfortunately, in low-birth-weight 
neonates, both rigid and flexible bronchoscopes may be too large in size to fit and these patients cannot tolerate spontaneous ventilation during these procedures, so it may not be possible to occlude the fistula in advance (3).

The endotracheal tube (ETT) should be positioned correctly considering the fistula diverting the inspiratory volume to stomach. The withdrawal of the ETT after advancing it deliberately towards right main bronchus can constitute a technique to correctly position the tube. While gently withdrawing the ETT, the left side is auscultated, ensuring that the bevel of the tube has been directed towards left main bronchus in order to detect the incoming air as soon as the tube tip is just above the carina below the fistula $(2,4)$. The shaft of the ETT may occlude the fistula, which is frequently on the posterior tracheal wall (2). This position of the ETT can be confirmed by using flexible bronchoscopy, which can also be used for evaluation of the fistula simultaneously instead of rigid bronchoscopy, a priori. The ETT should be secured at this level, while continuous assessment of the air entry to the left side may help to recognize the displacement immediately.

During the procedure, the arterial blood gas analysis allows the evaluation of adequate pulmonary ventilation, as well as, the peripheral oxygen saturation. Positive-pressure ventilation may be required in the presence of poorly compliant lungs like that of preterm infants (4). Especially during positive pressure ventilation, the air inflow may follow the path of fistula, which has the least resistance. The air inflow through the fistula may cause excessive distension of the stomach interfering with diaphragmatic movement resulting in atelectasis. This adverse event can be prevented by gastrostomy, however, gastrostomy has its own adverse events leading to complications such as gastric rupture. Moreover, gastrostomy may also constitute a bronchocutaneous fistula causing ineffective ventilation, resulting in a preference towards sparing the gastrostomy procedure for the neonates in imminent risk of gastric perforation $(1,4)$. The spontaneous ventilation technique should be considered in order to minimize the gastric insufflation (4). On the other hand, the presence of gastrostomy tube may help to confirm the placement of the ETT if an FOB is not available. The placement of the gastrostomy tube under water seal would reveal air bubbles, if the ETT is above the fistula, which requires a slight withdrawal and then advancement of the ETT until the bubbling ceases. The end-tidal carbon dioxide $\left(\mathrm{etCo}_{2}\right)$ monitoring may also help for the confirmation of the correct placement of the tube. If the analyser attached to the gastrostomy tube reveals a trace of etCO $\mathrm{CO}_{2}$, then it shows that the tube is above the fistula, so that, withdrawal followed by advancement of the tube should be performed (2).

\section{Maintenance}

The maintenance of anesthesia can be provided by either inhalational anesthetics or total intravenous anesthesia or both. The use of neuromuscular blocking agents are often needed for adequate muscle relaxation. A caudal or lumbar epidural catheter may reduce the use of general anesthetic agent requirements (5). The inspiratory fraction of oxygen in air/oxygen mixture should be titrated according to the $\mathrm{SpO}_{2}$, to keep the level $>95 \%$ in order to avoid hyperoxemia.

The customary right-sided posterolateral approach using thoracotomy from the $4-5^{\text {th }}$ intercostal spaces requires the neonate to be positioned on left-lateral side with pads under the thorax towards left armpit to facilitate the surgical access from the right-side (3). In this position the author's experience is to place the right arm above the head with a roll between the right arm, right shoulder and head. This position may be problematic to provide intraoperative warming, which should include forcedair warming blankets under the patient, covering all extremities and the head, and infusion of warm fluids to avoid hypothermia. The thoracoscopic approach has been presented as an alternative technique as experienced has been gained. In a modified left-lateral decubitus position, a $5 \mathrm{mmHg}$ capnothorax is provided to approach the fistula (5). The similar surgical position for both techniques requires verifying the correct placement of the tube tip using flexible pediatric bronchoscope, because the displacement of the tube tip during positioning results in gastric insufflation and difficulty in ventilation.

Until the division of the fistula is completed, maintenance of the communication between the surgeon and the anesthetist is crucial. The ventilation is a true challenge for the anesthetist, because it is most commonly complicated with hypercapnia and desaturation, as well as, complete inability to ventilate. Acute changes may occur due to surgical manipulations disturbing the placement of the ETT and the gas exchange. Short bursts should be performed in coordination with the anesthetist to provide 1-2 minutes of lung relaxation in order to improve critical levels of oxygen saturation $(3,5)$. The ventilation should be adjusted during lung retractions with meticulous attention to surgical site. Hence, manual ventilation would be more 
efficient to maintain careful adjustments and also more helpful to keep the cooperation between the anesthetist and the surgeon to maintain adequate oxygenation. Aside from the retractions occluding trachea and bronchi, the azygos vein often needs to be tied off to facilitate esophageal approach. This procedure requires an occlusion test with regard to evaluating its tolerability by the neonate (3). An arterial line allowing to monitor the real-time changes in blood pressure would be helpful during the operation.

In order to facilitate the location of proximal pouch, the anesthetist gently advances an indwelling oro-esophageal tube downwards and the dissection is continued upwards to achieve sufficient length for anastomosis. After completion of the anastomosis on the posterior wall, the oro-esophageal tube is frequently replaced with a smaller sized nasogastric (NG) feeding tube for the surgeon to discriminate the anterior and posterior walls of the esophagus. This feeding tube is to be maintained after the surgery to allow enteric feeding during the usual postoperative 1-week prior to the esophagogram to be performed. It is crucial to remember that this tube must be fixed in place due to a tendency for displacement (5).

During the thoracoscopic approach to the fistula, onelung ventilation technique can be used. While a tube that would fit into a small main stem bronchus may not be available, advancement of a Fogarty catheter into the right main bronchus may constitute an alternative technique (2). Both techniques require FOB guidance. The one-lung ventilation with 5-6 $\mathrm{mmHg} \mathrm{CO}_{2}$ insufflation to the right hemithorax may lead to hypercapnia and acidosis, as well as, hypoxia, which cannot be tolerated by small neonates (5). A sudden rise in etCO $\mathrm{CO}_{2}$ should be an alarming sign for the anesthetist to consider an injury in the collapsed lung or trapped insufflated gas. The coordination between the anesthetist and the surgeon optimizes the ventilation, because lower tidal volumes may be needed when the surgeons require less mediastinal movement to approach the fistula (2).

\section{Emergence}

The repair of the fistula may require expanding the previously atelectatic alveoli. In order to provide adequate ventilation following the repair, the ETT should remain for a period of time. The use of caudal or lumbar epidural catheter for analgesia during the operation constitutes an opioid-sparing technique, which facilitates early extubation. However, these patients, especially the ones with low birth weight $(<2,000 \mathrm{~g})$, tracheomalacia or poor lung functions, as well as, the ones exposed to secondary complications such as vocal cord paralysis, usually remain intubated postoperatively to avoid the complications of reintubation $(1,2,5)$. On the other hand, in thoracotomies, the retraction of the ipsilateral lung may result in contusions leading to an increase in oxygen and inspiratory pressure requirements, which can be overcome by mechanical ventilation during the first 12-24 hours postoperatively (2). Although early extubation is not frequently desired, opioid-sparing anesthetic and analgesic technique has proven to be beneficial, but eventually the timing of extubation should be decided by a multidisciplinary team including the surgeon, the anesthetist and the neonatologist/intensivist (5).

\section{Postoperative period}

Since these patients remain intubated for a period of time postoperatively, the neonatal intensive care unit (NICU) should be planned prior to the operation (2). However, this period of time is preferred to be as short as possible to protect the anastomosis from prolonged exposure to the pressure exerted by the ETT. On the other hand, these infants may often require reintubation due to secretions or tracheobronchomalacia. The reintubation process exposes the neonate to hyperextension of the neck causing stretch in the anastomosis line, which is to be protected during this period. Since reintubation is not a desired intervention, the timing for extubation of the trachea should be decided by a team including the anesthetist, surgeon and neonatologist/ intensivist $(4,5)$.

The fluid and electrolyte requirements should be maintained according to hemodynamic parameters and laboratory tests including serum sodium, creatinine and urea (2).

Maintenance of normothermia during the operation may reduce the incidence of adverse events intraoperatively, leading to a lower risk of hypothermia, apnea and metabolic acidosis in the postoperative period. However, pneumothorax, tracheal leak, laryngeal nerve injury and pneumonia may all occur in the postoperative period. Arterial blood gas analysis and the total blood count focusing on hematocrit level should be considered during this period (5).

An epidural catheter may provide adequate pain management, however, regional techniques may be better to be avoided in neonates with cardiac anomalies due to a decrease in systemic vascular resistance. As alternative 
analgesic techniques, opioid infusions and/or wound infiltration techniques may also be used (2).

\section{Conclusions}

The anesthetic management of neonates with TEF with or without EA, should mainly focus on the airway management and ventilation strategies. The maintenance of the patency of the airway and adequate ventilation and oxygenation depends on the communication between the pediatric surgeon and the anesthetist throughout the surgery. Aside from the airway and respiratory problems, the anesthetic considerations should also include the optimization of the status pertaining to co-morbidities due to associated congenital anomalies, which increase the incidence of adverse events, complications and morbidity in the perioperative period. The neonates are preferred to remain endotracheally intubated for a period of time postoperatively, hence, an NICU bed should be organized for these neonates prior to definitive repair.

\section{Acknowledgments}

Funding: None.

\section{Footnote}

Provenance and Peer Review: This article was commissioned by the Guest Editor (Tutku Soyer) for the series "Tracheoesophageal Fistula" published in Current Challenges in Thoracic Surgery. The article has undergone external peer review.

Conflicts of Interest: The author has completed the ICMJE uniform disclosure form (available at https://ccts. amegroups.com/article/view/10.21037/ccts-20-183/coif). The series "Tracheoesophageal Fistula" was commissioned by the editorial office without any funding or sponsorship. The author has no other conflicts of interest to declare.

Ethical Statement: The author is accountable for all aspects of the work in ensuring that questions related to the accuracy or integrity of any part of the work are appropriately investigated and resolved.

Open Access Statement: This is an Open Access article distributed in accordance with the Creative Commons Attribution-NonCommercial-NoDerivs 4.0 International
License (CC BY-NC-ND 4.0), which permits the noncommercial replication and distribution of the article with the strict proviso that no changes or edits are made and the original work is properly cited (including links to both the formal publication through the relevant DOI and the license). See: https://creativecommons.org/licenses/by-nc-nd/4.0/.

\section{References}

1. Hunt RW, Perkins EJ, King S. Peri-operative management of neonates with oesophageal atresia and tracheooesophageal fistula. Paediatr Respir Rev 2016;19:3-9.

2. Edelman B, Selvaraj BJ, Joshi M, et al. Anesthesia Practice: Review of Perioperative Management of H-Type Tracheoesophageal Fistula. Anesthesiol Res Pract 2019;2019:8621801.

3. Kinottenbelt G, Skinner A, Seefelder C. Tracheooesophageal fistula (TOF) and oesophageal atresia (OA). Best Pract Res Clin Anaesthesiol 2010;24:387-401.

4. Bachiller PR, Chou JH, Romanelli TM, et al. Chapter 36. Neonatal Emergencies. In, Cote and Lerman's A Practice of Anesthesia for Infants and Children. Ed's. Cote CJ, Lerman J, Anderson BJ. 5th ed. Elsevier Saunders; 2013:746-65.

5. Wall J, Albanese C, Claure RE, et al. Chapter 12.5. Pediatric General Surgery. In, Anesthesiologist's Manual of Surgical Procedures Ed. Jaffe RA. Schmiesing CA, Golianu B. 5th ed. Wolters Kluwer Health; 20141287-92.

6. Sharma N, Srinivas M. Laryngotracheobronchoscopy prior to esophageal atresia and tracheoesophageal fistula repair-its use and importance. J Pediatr Surg 2014;49:367-9.

7. Hseu A, Recko T, Jennings R, et al. Upper Airway Anomalies in Congenital Tracheoesophageal Fistula and Esophageal Atresia Patients. Ann Otol Rhinol Laryngol 2015;124:808-13.

8. Londahl M, Irace AL, Kawai K, et al. Prevalence of Laryngeal Cleft in Pediatric Patients With Esophageal Atresia. JAMA Otolaryngol Head Neck Surg 2018;144:164-8.

9. Fraga JC, Adil EA, Kacprowicz A, et al. The association between laryngeal cleft and tracheoesophageal fistula: myth or reality? Laryngoscope 2015;125:469-74.

doi: $10.21037 /$ ccts-20-183

Cite this article as: Uzumcugil F. Anesthetic management of tracheo-esophageal fistula. Curr Chall Thorac Surg 2022;4:27. 